\title{
Twelve weeks supplementation with an extended-release caffeine and ATP- enhancing supplement may improve body composition without affecting hematology in resistance-trained men
}

Jordan M. Joy ${ }^{1 *}$, Roxanne M. Vogel ${ }^{1}$, Jordan R. Moon ${ }^{2}$, Paul H. Falcone ${ }^{3}$, Matt M. Mosman ${ }^{4}$ and Michael P. Kim³

\begin{abstract}
Background: Increased ATP levels may enhance training-induced muscle accretion and fat loss, and caffeine is a known ergogenic aid. A novel supplement containing ancient peat and apple extracts has reported enhanced mitochondrial ATP production and it has been coupled with an extended-release caffeine. Therefore, the purpose of this investigation was to determine the effects of this supplement on body composition when used in conjunction with 12 weeks of resistance training.

Methods: Twenty-one resistance-trained subjects $(27.2 \pm 5.6 \mathrm{y} ; 173.5 \pm 5.7 \mathrm{~cm} ; 82.8 \pm 12.0 \mathrm{~kg})$ completed this study. Subjects supplemented daily with either 1 serving of the supplement (TRT), which consisted of $150 \mathrm{mg}$ ancient peat and apple extracts, $180 \mathrm{mg}$ blend of caffeine anhydrous and pterostilbene-bound caffeine, and $38 \mathrm{mg} \mathrm{B}$ vitamins, or an equal-volume, visually-identical placebo (PLA) 45 min prior to training or at the same time of day on rest days. Supervised resistance training consisted of 8 weeks of daily undulating periodized training followed by a 2-week overreach and a 2-week taper phase. Body composition was assessed using DEXA and ultrasound at weeks 0, 4, 8, 10, and 12. Vital signs and blood markers were assessed at weeks 0, 8, and 12.

Results: Significant group $x$ time $(p<0.05)$ interactions were present for cross-sectional area of the rectus femoris, which increased in TRT $\left(+1.07 \mathrm{~cm}^{2}\right)$ versus PLA $\left(-0.08 \mathrm{~cm}^{2}\right)$, as well as muscle thickness (TRT: $+0.49 \mathrm{~cm} ;$ PLA: $\left.+0.04 \mathrm{~cm}\right)$. A significant group $x$ time $(p<0.05)$ interaction existed for creatinine (TRT: $+0.00 \mathrm{mg} / \mathrm{dL} ; \mathrm{PLA}:+0.15 \mathrm{mg} / \mathrm{dL}$ ) and estimated glomerular filtration rate (TRT: $-0.70 \mathrm{~mL} / \mathrm{min} / 1.73 ; \mathrm{PLA}:-14.6 \mathrm{~mL} / \mathrm{min} / 1.73$ ), which remained within clinical ranges, but no other significant observations were observed.

Conclusions: Supplementation with a combination of extended-release caffeine and ancient peat and apple extracts may enhance resistance training-induced skeletal muscle hypertrophy without adversely affecting blood chemistry.
\end{abstract}

Keywords: Ergogenic aid, Hypertrophy, Safety, Elevatp, Purenergy

\footnotetext{
* Correspondence: jmjoyx@gmail.com

'Department of Nutrition and Food Sciences, Texas Woman's University, 401

AME Drive \#7101, Denton, TX 76207, USA

Full list of author information is available at the end of the article
} 


\section{Background}

Adenosine-5'-triphosphate (ATP) and ATP metabolites are involved in a myriad of biological processes including cardiac function, neurotransmission, blood flow, and muscle contraction [2, 26], and it is strongly suggested that increased ATP levels correlate with improved health and performance $[18,23,40]$. Direct supplementation with ATP seems to be effective for increasing ATP when measured in whole blood [23], but there have been mixed results $[4,11]$. Therefore, an indirect approach for increasing ATP levels may be desirable. Previously, ReyesIzquierdo and colleagues determined that a $150 \mathrm{mg}$ dose of a blend of ancient peat and apple extracts significantly increased blood ATP compared to placebo in 18 [31] and 20 [32] subjects. In the latter research, blood ATP increased by $40 \%$ at $60 \mathrm{~min}$ following ingestion, which dropped to $28 \%$ at $120 \mathrm{~min}$ following ingestion. A muscle biopsy was conducted in one subject, and ATP levels were observed to increase in muscle tissue by $281 \%$ at $60 \mathrm{~min}$ and $433 \%$ at 120 min following ingestion [32]. Preliminary reports from this laboratory also support an increase in blood ATP levels, and suggest this occurs without an increase in reactive oxygen species, which may be associated with increased ATP production [12]. In fact, ancient peat and apple extracts may actually decrease reactive oxygen species [31], possibly blunting the increase caused by resistance training [3].

Previous reports on indirect ATP enhancement are sparse. However, the beneficial effects of ancient peat and apple extracts have recently been shown to positively enhance muscle mass [24]. Moreover, direct ATP supplementation may be efficacious for positively augmenting performance $[23,30,44]$ and body composition [44]. Wilson et al. reported increases in whole-body lean mass as well as quadriceps muscle thickness in the ATPsupplemented group compared to placebo following a 12-week resistance training protocol.

There are a multitude of studies on the ergogenic properties of caffeine, which has been demonstrated to improve endurance [14], high-intensity sport [36], strength [8], and cognitive [27] performance. Moreover, caffeine may enhance body composition by way of increased metabolism. Caffeine has been demonstrated to increase fatty acid oxidation, energy expenditure, and thermogenesis [5, 17, 37], possibly via increased epinephrine, which can up-regulate both lipolysis and glycogenolysis, and is consistent with reports on caffeine supplementation and high-intensity exercise [21]. Astrup et al. has reported that caffeine supplementation increases vascular smooth muscle tone, another paralleled effect of epinephrine. These researchers also observed an increase in energy expenditure that correlated with plasma triglyceride levels [5]. Increased utilization of free fatty acids with caffeine supplementation has been reported in both overweight and normal weight individuals [1]. Furthermore, caffeine supplementation has been confirmed to reduce body weight and body fat in obese rats [39]. However, the chronic effects of caffeine supplementation on body fat in healthy humans are poorly understood.

While direct and indirect ATP supplementation may be capable of augmenting resistance training induced changes in body composition, minimal research exists on this topic. Even less is known when ancient peat and apple extracts are coupled with other ingredients. In addition, caffeine has been noted to increase metabolism as well as acute exercise performance. When used in conjunction with elevated ATP levels, it may be possible to simultaneously increase lean muscle mass, while reducing adiposity. Therefore, the primary purpose of this study was to assess the effects of a proprietary blend of ancient peat and apple extracts coupled with an extended-release caffeine on changes in body composition following 12 weeks of resistance training. The secondary purpose of this study was to assess the safety of the two ingredients. It was hypothesized that the supplement will aid increases in lean muscle mass and muscle hypertrophy and decrements in body fat without changing hematological safety markers.

\section{Methods \\ Participants}

Twenty-one healthy, resistance-trained, male subjects $(27.2 \pm 5.6 y ; 173.5 \pm 5.7 \mathrm{~cm} ; 82.8 \pm 12.0 \mathrm{~kg})$ completed the current investigation. Thirty-three subjects were recruited, and 3 subjects did not complete the study due to scheduling conflicts, 3 were not compliant with protocols, and 2 sustained injuries during the study unrelated to training or supplementation. Each subject was required to be capable of lifting 1.5 times their bodyweight in the squat and deadlift and 1 times bodyweight in the bench press. At baseline, the placebo (PLA) group was able to squat 1.71 \pm 0.21 , bench press $1.45 \pm 0.19$, and deadlift $2.17 \pm 0.25$ times their bodyweight, and the treatment (TRT) group was able to squat $1.58 \pm 0.20$, bench press $1.33 \pm 0.20$, and deadlift $1.97 \pm 0.26$ times their bodyweight. Approval for research with human subjects was obtained from the MusclePharm Sports Science Institute IRB, and subjects provided written informed consent documents prior to participation in the study.

\section{Experimental design}

The precise methods of the present study have previously been published with the exception of a difference in treatment [24]. Subjects were randomly assigned to either the PLA $(n=11)$ or TRT $(n=10)$ groups. They were instructed to consume 1 serving $(2 \mathrm{~mL})$ of either PLA or TRT (150 mg ElevATP ${ }^{\mathrm{m} w}$, FutureCeuticals Inc., Momence, IL; $180 \mathrm{mg}$ blend of caffeine anhydrous and PurEnergy ${ }^{\text {tm }}$, Chromadex Inc., Irvine, CA; and $38 \mathrm{mg} \mathrm{B}$ vitamins) 
45 min prior to training on training days or at a similar time of day on rest days. For a detailed composition of ElevATP $^{\mathrm{ms}}$, composed of ancient peat and apple extracts, see [31]. The PurEnergy ${ }^{\mathrm{Tm}}$ ingredient is composed of $43 \%$ caffeine and $57 \%$ pterostilbene. Total caffeine content per serving was $\sim 129 \mathrm{mg}$. Supplement vials were weighed to ensure compliance. Subjects were resistance trained under the guidance of a certified strength and conditioning specialist 3 days per week for 8 weeks followed by a 2 -week overreach and 2-week taper phase corresponding to weeks 9-10 and 11-12, respectively. A eucaloric diet consisting of $50 \%$ calories from carbohydrates, $25 \%$ from protein, and $25 \%$ from fat was prescribed to all subjects at the onset of the study, and diets were tracked weekly via 3-day food logs. Total calories were determined for each individual based on the Mifflin St. Jeor equation adjusted for activity level. Subjects were measured at weeks 0, 4, 8, 10, and 12 for all body composition variables. Blood draws and vital sign measurements were conducted at weeks 0 , 8 , and 12 . Body composition variables collected consisted of DEXA, which determined lean soft tissue (LST), fat mass (FM), and body fat percentage (\% Fat), and ultrasound, which determined cross-sectional area (CSA), muscle thickness (MT), and fat thickness (FT).

\section{Resistance training program}

Weeks 1-8 consisted of one muscle hypertrophy-oriented workout, one power workout, and one strength-oriented workout each week. The hypertrophy session consisted of barbell back squat, bench press, deadlift, incline bench press, hammer strength power squat machine, hammer strength isolateral bench press, leg press, leg extension, leg curl, and triceps extension performed for 3 sets of 6-12 repetition at $60-80 \% 1 \mathrm{RM}$ intensity. The power session consisted of barbell back squat, bench press, and deadlift exercises performed for 5 sets of 2-5 repetitions with a goal of high velocity of movement with 40-60 \% 1RM intensity. After performing the main exercises on the power day, subjects performed bent over row, pulldown, dumbbell row, shoulder press, lateral raise, and bicep curl exercises for the goal of muscle hypertrophy as described for chest and leg exercises. The strength session consisted of barbell back squat, bench press, deadlift, shoulder press, and pulldown exercises performed for 3 sets of 1-5 repetitions at 85$100 \% 1 \mathrm{RM}$ intentisty. Following the resistance exercises on the strength day, participants performed 2-6 sets of 10-30s Wingates on a cycle ergometer with $2-4$ min rest. Participants rested 48-72 $\mathrm{h}$ between each training day, and 30$120 \mathrm{~s}$ between sets on the hypertrophy day or 2-5 min between sets on the power and strength days. All exercises were completed within 60-120 min. During the overreach phase, participants performed high-volume workouts, similar to the hypertrophy-oriented workouts performed during weeks $1-8$, on Monday through Thursday, with a strength- oriented workout or performance testing conducted on Friday for weeks 9 and 10, respectively. The taper weeks consisted of one power session on Mondays. On Wednesdays and Fridays, participants performed 1-3 heavy sets for 2-5 repetitions of the back squat, bench press, and deadlift, and each heavy exercise was immediately followed by the same exercise for 3 power-oriented sets, as previously described, before progressing to the next exercise.

\section{Measurements}

Urine specific gravity was determined on each body composition testing day to ensure measurements were conducted in a euhydrated state. On 3 occasions, a participant was required to drink water until another urine sample could be submitted and verified for adequate hydration status. Body weight was determined using a calibrated column scale (SECA, Chino, CA). Body composition was analyzed for whole-body and segmental LST, FM, and \% Fat using DEXA (Lunar Prodigy Primo, General Electric, Fairfield, CN) with enCORE software (Version 15, Madison, WN). Testretest reliability for DEXA LST, \% Fat, and FM, as measured using 15 subjects, resulted in an average ICC of $>0.99$. CSA, MT, and FT were determined using ultrasound (Logiq e, General Electric, Fairfield, $\mathrm{CN})$. The minimum differences [43] needed to be considered a true change are $0.107 \mathrm{~cm}^{2}$ and $0.038 \mathrm{~cm}$ for CSA and MT, respectively. Ultrasonography determined CSA was measured at $75 \%$ femur length, as defined as the distance from the anterior superior iliac spine to the superior aspect of the patella. MT of the quadriceps was measured at $50 \%$ femur length, defined as the distance from the greater trochanter of the femur to the lateral epicondyle of the femur. MT was defined as the combined thickness of the vastus lateralis and vastus intermedius. The distance from the superficial aspect the femur to the deep aspect of the superficial fascia of the vastus lateralis was measured. FT was measured at the same site as MT, and it was defined as the distance from the superficial aspect of the vastus lateralis fascial layer to the deep aspect of the hypodermis. For MT, FT, and CSA, ICC was 0.99, 0.99 , and 0.97, respectively. Vital signs were determined using an automated, digital sphygmomanometer (Omron Corporation, Kyoto, Japan). Blood draws were performed via venipuncture by a trained phlebotomist. Following a 10-h fast, all subjects submitted a blood sample for analysis in the morning to control for diurnal variations. Blood variables consisted of white blood cell count (WBC), red blood cell count (RBC), hemoglobin, hematocrit, mean corpuscular volume (MCV), mean corpuscular hemoglobin $(\mathrm{MCH})$, mean corpuscular hemoglobin concentration $(\mathrm{MCHC})$, red blood cell distribution width (RDW), platelets (absolute), neutrophils 
(percent and absolute), lymphocytes (percent and absolute), monocytes (percent and absolute), eosinophils (percent and absolute), basophils (percent and absolute), serum glucose, blood urea nitrogen (BUN), creatinine, estimated glomerular filtration rate (eGFR), BUN:creatinine, sodium, potassium, chloride, carbon dioxide, calcium, protein, albumin, globulin, albumin:globulin (A/G), bilirubin, alkaline phosphatase, aspartate aminotransferase (AST), alanine aminotransferase (ALT), total cholesterol, triglycerides, high density lipoprotein (HDL) cholesterol, very low density lipoprotein (VLDL), and low density lipoprotein (LDL) cholesterol. Blood variables were analyzed by a third party (Laboratory Corporation of America, Denver, CO). Intertest reliability results from 12 men and women measured up to one week apart at the aforementioned laboratory resulted in no significant differences from day-to-day $(p>$ 0.05) and an average inter-test Coefficient of Variation of $6.9 \%$ for all tests.

\section{Statistical analyses}

Repeated measures ANOVAs were performed to assess group, time, and group by time interactions with a significant $p$-value considered as $\leq 0.05$. A Bonferroni posthoc analysis was used to locate differences. Independent T-tests were conducted on the delta values for each time point. Dependent T-tests were conducted to determine within group differences for all body composition and hematology data with a significant interaction. Statistica

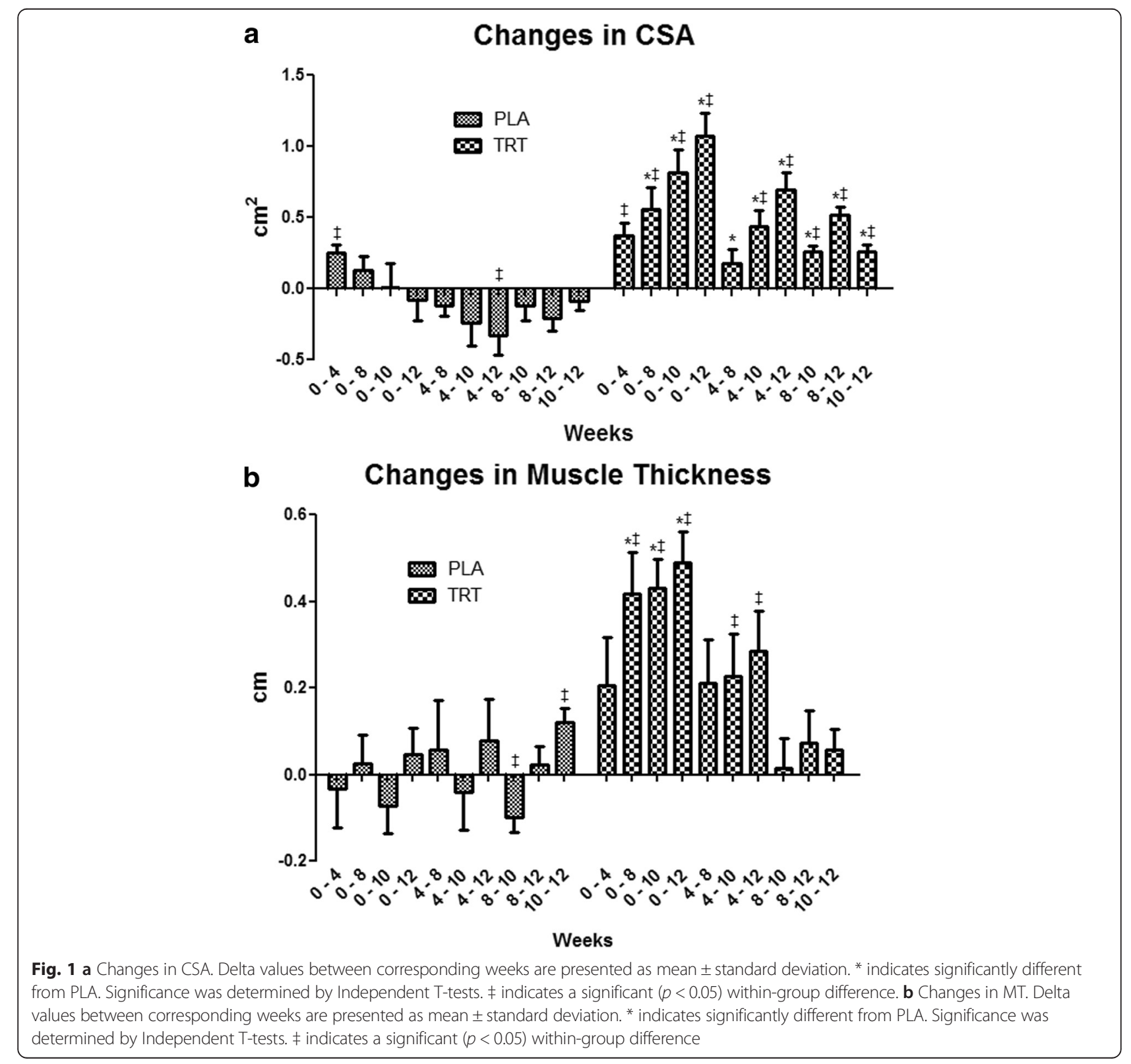


(Version 10, Statsoft, Tulsa, OK) was used for all statistical analyses.

\section{Results}

Significant time and group by time $(p<0.05)$ interactions were present for CSA (Fig. 1a, Fig. 2, and Table 1). CSA was greater in TRT versus PLA at weeks 8, 10, and 12 . Moreover, CSA increased in TRT compared to PLA between all time points except for between weeks 0 and 4 using independent T-tests $(p<0.05)$. There were significant time and group by time $(p<0.05)$ interactions observed for MT (Fig. 1b, Table 1). MT increased to a greater extent in TRT than PLA from pre to weeks 8 , 10 , and 12 . There was a significant group by time $(p<$ 0.05 ) interaction for both left and right leg FM and \% Fat, which decreased in TRT versus PLA (Table 2). Left and right leg \% Fat did not reach significance in the post hoc analysis, yet independent T-tests conducted on the delta values revealed a significant difference between week 0 and weeks 4,8 , and 10 (Table 2). A significant main effect for time $(p<0.05)$ was found for body weight, LST, and LST of the arms, legs, and trunk, but no significant group by time interactions existed for these variables (Table 1 ). Moreover, a significant main effect for time $(p<0.05)$ was observed for FT, FM, and \% Fat, yet no significant group by time interactions were observed for
FM or \% Fat of the arms or trunk (Table 2). No differences were observed for average daily calories, carbohydrates, fats, or proteins consumed each week throughout the study $(p>0.05)$.

No changes were observed for systolic or diastolic blood pressure or heart rate. A significant group by time $(p<0.05)$ interaction was present for creatinine, which increased in PLA from pre to week 12 (TRT: 0.00 ; PLA: $+0.15 \mathrm{mg} / \mathrm{dL}$ ) and from week 8 to week 12 (TRT: -0.05 ; PLA: $+0.09 \mathrm{mg} / \mathrm{dL}$ ). There was a significant group by time $(p<0.05)$ interaction present for eGFR, which decreased in PLA from pre to week 12 (TRT: -0.70 ; PLA: $-14.6 \mathrm{~mL} / \mathrm{min} / 1.73$ ) and from week 8 to week 12 (TRT: +5.10; PLA: $-7.73 \mathrm{~mL} /$ $\mathrm{min} / 1.73)$. No other significant interactions were observed for any safety markers, and each marker remained within the physiological reference range (Tables 3, 4, and 5).

\section{Discussion}

The results of this study support the hypotheses. Although no interactions were observed for any DEXAbased measurements of LST between groups, both ultrasound-based measurements of CSA and MT increased while lower body measures of FM and \% Fat decreased. No abnormal changes in vital signs or blood markers were detected. Creatinine and eGFR

Table 1 Lean Body Composition Data. Data are presented as mean \pm standard deviation

\begin{tabular}{|c|c|c|c|c|c|c|c|}
\hline Variable & Group & Pre & Week 4 & Week 8 & Week 10 & Post & $p$ \\
\hline \multirow[t]{2}{*}{$\overline{\mathrm{CSA}}\left(\mathrm{cm}^{2}\right)$} & PLA & $3.60 \pm 1.57$ & $3.85 \pm 1.49^{a}$ & $3.73 \pm 1.32$ & $3.61 \pm 1.27$ & $3.52 \pm 1.39^{b c}$ & $<0.001$ \\
\hline & TRT & $3.75 \pm 1.15$ & $4.13 \pm 1.14^{\mathrm{a}}$ & $4.31 \pm 1.16^{* a}$ & $4.56 \pm 1.09^{* a b c}$ & $4.82 \pm 1.10^{* a b c d}$ & \\
\hline \multirow[t]{2}{*}{ MT (cm) } & PLA & $5.50 \pm 0.72$ & $5.47 \pm 0.50$ & $5.53 \pm 0.74$ & $5.43 \pm 0.66^{c}$ & $5.55 \pm 0.68^{d}$ & $<0.001$ \\
\hline & TRT & $5.33 \pm 0.63$ & $5.54 \pm 0.73$ & $5.75 \pm 0.64^{* a}$ & $5.77 \pm 0.61^{* a b}$ & $5.82 \pm 0.54^{* a b}$ & \\
\hline \multirow[t]{2}{*}{ LST (kg) } & PLA & $65.3 \pm 8.1$ & $65.2 \pm 6.5$ & $65.7 \pm 6.6$ & $66.2 \pm 6.8$ & $65.5 \pm 6.9$ & 0.62 \\
\hline & TRT & $62.3 \pm 8.5$ & $63.3 \pm 8.7$ & $63.5 \pm 8.7$ & $64.1 \pm 8.4$ & $63.4 \pm 8.5$ & \\
\hline \multirow[t]{2}{*}{ Body Weight (kg) } & PLA & $83.7 \pm 10.5$ & $86.3 \pm 10.6$ & $86.1 \pm 11.5$ & $87.3 \pm 12.1$ & $86.4 \pm 11.7$ & 1.00 \\
\hline & TRT & $81.8 \pm 14.0$ & $84.0 \pm 13.7$ & $84.1 \pm 15.0$ & $84.9 \pm 15.5$ & $84.6 \pm 16.4$ & \\
\hline \multirow[t]{2}{*}{ R Leg LST (kg) } & PLA & $10.6 \pm 1.7$ & $10.9 \pm 1.9$ & $10.9 \pm 1.5$ & $11.2 \pm 1.7$ & $10.9 \pm 1.3$ & 0.39 \\
\hline & TRT & $10.3 \pm 1.5$ & $10.5 \pm 1.6$ & $10.6 \pm 1.5$ & $10.6 \pm 1.6$ & $10.8 \pm 1.7$ & \\
\hline \multirow[t]{2}{*}{ L Leg LST (kg) } & PLA & $10.5 \pm 1.5$ & $10.8 \pm 1.7$ & $10.8 \pm 1.4$ & $11.1 \pm 1.6$ & $10.7 \pm 1.1$ & 0.35 \\
\hline & TRT & $10.2 \pm 1.4$ & $10.6 \pm 1.5$ & $10.8 \pm 1.6$ & $10.7 \pm 1.6$ & $10.7 \pm 1.7$ & \\
\hline \multirow[t]{2}{*}{ R Arm LST (kg) } & PLA & $4.7 \pm 0.6$ & $4.5 \pm 0.5$ & $4.3 \pm 0.5$ & $4.2 \pm 0.5$ & $4.1 \pm 0.5$ & 0.84 \\
\hline & TRT & $4.4 \pm 0.8$ & $4.2 \pm 0.7$ & $3.9 \pm 0.5$ & $3.8 \pm 0.5$ & $3.7 \pm 0.5$ & \\
\hline \multirow[t]{2}{*}{ L Arm LST (kg) } & PLA & $4.5 \pm 0.5$ & $4.5 \pm 0.4$ & $4.1 \pm 0.5$ & $4.0 \pm 0.4$ & $4.0 \pm 0.5$ & 0.61 \\
\hline & TRT & $4.3 \pm 0.7$ & $4.1 \pm 0.6$ & $3.8 \pm 0.5$ & $3.7 \pm 0.5$ & $3.7 \pm 0.4$ & \\
\hline \multirow[t]{2}{*}{ Trunk LST (kg) } & PLA & $30.8 \pm 4.3$ & $30.2 \pm 2.9$ & $31.2 \pm 3.4$ & $31.5 \pm 3.5$ & $31.6 \pm 4.6$ & 0.27 \\
\hline & TRT & $29.2 \pm 5.6$ & $29.9 \pm 4.9$ & $30.3 \pm 4.8$ & $31.3 \pm 4.6$ & $30.5 \pm 4.6$ & \\
\hline
\end{tabular}

*indicates significantly different from PLA at the corresponding time point. The p-value is derived from an ANOVA and representative of a main effect for group by time. Significant within-group time differences are indicated by ${ }^{a}$ (different from pre), ${ }^{b}$ (different from week 4$)$, ${ }^{c}\left(\right.$ different from week 8 ), and ${ }^{\mathrm{d}}$ (different from week 10) for variables with a significant group $x$ time interaction 
Table 2 Adipose Body Composition Data. Data are presented as mean \pm standard deviation

\begin{tabular}{|c|c|c|c|c|c|c|c|}
\hline Variable & Group & Pre & Week 4 & Week 8 & Week 10 & Post & $p$ \\
\hline \multirow[t]{2}{*}{ FT $(\mathrm{cm})$} & PLA & $0.52 \pm 0.20$ & $0.49 \pm 0.19$ & $0.53 \pm 0.19$ & $0.48 \pm 0.18$ & $0.53 \pm 0.16$ & 0.96 \\
\hline & TRT & $0.64 \pm 0.43$ & $0.63 \pm 0.46$ & $0.66 \pm 0.43$ & $0.62 \pm 0.39$ & $0.64 \pm 0.43$ & \\
\hline \multirow[t]{2}{*}{$\mathrm{FM}(\mathrm{kg})$} & PLA & $16.7 \pm 5.6$ & $18.6 \pm 6.3$ & $18.4 \pm 6.6$ & $18.5 \pm 6.9$ & $18.5 \pm 6.5$ & 0.17 \\
\hline & TRT & $17.4 \pm 9.4$ & $17.8 \pm 9.3$ & $18.3 \pm 10.5$ & $18.0 \pm 10.4$ & $18.8 \pm 10.9$ & \\
\hline \multirow[t]{2}{*}{$\%$ Fat } & PLA & $20.1 \pm 5.4$ & $21.8 \pm 5.5$ & $21.4 \pm 5.8$ & $21.3 \pm 5.9$ & $21.6 \pm 5.7$ & 0.14 \\
\hline & TRT & $21.0 \pm 8.8$ & $21.2 \pm 8.5$ & $21.4 \pm 9.4$ & $20.9 \pm 8.9$ & $21.7 \pm 9.3$ & \\
\hline \multirow[t]{2}{*}{ R Leg FM (kg) } & PLA & $2.5 \pm 0.7$ & $2.8 \pm 0.9^{a}$ & $2.8 \pm 0.9^{a}$ & $2.8 \pm 0.9^{a}$ & $2.7 \pm 0.8^{a}$ & 0.003 \\
\hline & TRT & $2.8 \pm 1.5$ & $2.8 \pm 1.4^{*}$ & $2.8 \pm 1.5^{*}$ & $2.6 \pm 1.3^{*}$ & $2.9 \pm 1.5^{d}$ & \\
\hline \multirow[t]{2}{*}{ L Leg FM (kg) } & PLA & $2.4 \pm 0.7$ & $2.8 \pm 0.9^{\mathrm{a}}$ & $2.7 \pm 0.9^{a}$ & $2.7 \pm 0.9^{a}$ & $2.7 \pm 0.8^{\mathrm{a}}$ & 0.01 \\
\hline & TRT & $2.8 \pm 1.5$ & $2.8 \pm 1.4^{*}$ & $2.8 \pm 1.5^{*}$ & $2.7 \pm 1.4^{*}$ & $2.9 \pm 1.5^{\mathrm{d}}$ & \\
\hline \multirow[t]{2}{*}{ R Arm FM (kg) } & PLA & $0.66 \pm 0.21$ & $0.70 \pm 0.22$ & $0.61 \pm 0.18$ & $0.61 \pm 0.20$ & $0.61 \pm 0.19$ & 0.30 \\
\hline & TRT & $0.72 \pm 0.43$ & $0.71 \pm 0.39$ & $0.62 \pm 0.42$ & $0.57 \pm 0.34$ & $0.59 \pm 0.37$ & \\
\hline \multirow[t]{2}{*}{ L Arm FM (kg) } & PLA & $0.64 \pm 0.21$ & $0.70 \pm 0.22$ & $0.58 \pm 0.18$ & $0.59 \pm 0.20$ & $0.60 \pm 0.19$ & 0.18 \\
\hline & TRT & $0.71 \pm 0.42$ & $0.71 \pm 0.39$ & $0.62 \pm 0.42$ & $0.56 \pm 0.33$ & $0.58 \pm 0.37$ & \\
\hline \multirow[t]{2}{*}{ Trunk FM (kg) } & PLA & $9.9 \pm 4.2$ & $11.0 \pm 4.2$ & $11.2 \pm 4.7$ & $11.3 \pm 4.8$ & $11.4 \pm 4.9$ & 0.62 \\
\hline & TRT & $9.8 \pm 5.4$ & $10.2 \pm 5.6$ & $10.8 \pm 6.5$ & $11.0 \pm 7.0$ & $11.3 \pm 7.1$ & \\
\hline \multirow[t]{2}{*}{ R Leg \% Fat } & PLA & $18.8 \pm 4.2$ & $20.2 \pm 4.6^{a}$ & $19.9 \pm 4.7$ & $19.4 \pm 4.5$ & $19.7 \pm 4.6$ & 0.03 \\
\hline & TRT & $20.6 \pm 8.1$ & $20.4 \pm 7.8^{\dagger}$ & $20.2 \pm 8.2^{\dagger}$ & $19.4 \pm 7.5^{\mathrm{tab}}$ & $20.3 \pm 7.7^{d}$ & \\
\hline \multirow[t]{2}{*}{ L Leg \% Fat } & PLA & $18.8 \pm 4.2$ & $20.2 \pm 4.6^{a}$ & $19.9 \pm 4.7$ & $19.4 \pm 4.6$ & $19.7 \pm 4.6$ & 0.03 \\
\hline & TRT & $20.6 \pm 8.1$ & $20.3 \pm 7.8^{\dagger}$ & $20.1 \pm 8.2^{\dagger}$ & $19.4 \pm 7.5^{\text {ta }}$ & $20.3 \pm 7.7^{d}$ & \\
\hline \multirow[t]{2}{*}{ R Arm \% Fat } & PLA & $12.3 \pm 3.6$ & $13.2 \pm 3.3$ & $12.3 \pm 3.0$ & $12.5 \pm 3.5$ & $12.9 \pm 3.4$ & 0.37 \\
\hline & TRT & $14.1 \pm 7.6$ & $14.5 \pm 7.7$ & $13.6 \pm 7.9$ & $13.2 \pm 7.6$ & $13.5 \pm 8.0$ & \\
\hline \multirow[t]{2}{*}{ L Arm \% Fat } & PLA & $12.3 \pm 3.6$ & $13.3 \pm 3.3$ & $12.3 \pm 2.9$ & $12.5 \pm 3.5$ & $12.9 \pm 3.4$ & 0.35 \\
\hline & TRT & $14.1 \pm 7.7$ & $14.6 \pm 7.7$ & $13.6 \pm 7.9$ & $13.1 \pm 7.6$ & $13.5 \pm 8.0$ & \\
\hline \multirow[t]{2}{*}{ Trunk \% Fat } & PLA & $23.8 \pm 7.4$ & $26.1 \pm 7.5$ & $25.5 \pm 7.8$ & $25.5 \pm 8.0$ & $25.5 \pm 7.5$ & 0.18 \\
\hline & TRT & $24.1 \pm 10.1$ & $24.3 \pm 9.6$ & $24.9 \pm 11.0$ & $24.4 \pm 10.4$ & $25.2 \pm 11.0$ & \\
\hline
\end{tabular}

*indicates significantly different from PLA at the corresponding time point. The $p$-value is derived from an ANOVA and representative of a main effect for group by time. $\dagger$ indicates significantly different from PLA at week 0 as determined by independent T-tests of the delta values. Significant within-group time differences are indicated by ${ }^{a}$ (different from pre), ${ }^{b}$ (different from week 4), ${ }^{c}$ (different from week 8 ), and ${ }^{d}$ (different from week 10 ) for variables with a significant group $x$ time interaction

changed to a greater extent in PLA compared to TRT, indicating a normal variation in these markers. Furthermore, every blood marker remained within the accepted physiological range. With these considerations, it is unlikely that these changes were produced by supplementation.

Previous reports on direct and indirect ATP supplementation are in agreement with the present results. While there were no significant interactions reported for measures of body fat, Wilson et al. [44] observed significant increases in quadriceps muscle thickness in ATP-supplemented participants versus placebo following 12 weeks of periodized resistance training. There was also a significant increase in whole-body lean mass between groups over time, which only increased over time in the present study. Moreover, the present results concerning muscle mass are consistent with the observations of supplementation with ancient peat and apple extracts without caffeine [24]. Wilson et al. [44] reported no interactions for all measured blood markers, while the present study observed a possible effect for creatinine and eGFR, yet the relevance of these observations may be undue. This is also in agreement with Coolen and colleagues [13] who observed no changes in blood markers following 4 weeks direct supplementation with $5 \mathrm{~g} /$ day of ATP. Furthermore, multi-ingredient products containing caffeine have previously been reported to be safe for human consumption as determined by changes in hematology and hemodynamics, but these studies were of relatively short duration (2-4 weeks) compared to the present study $[16,42]$.

The blend of ancient peat and apple extracts may be capable of promoting skeletal muscle hypertrophy by 
Table 3 Vital Signs and Blood Lipid Data

\begin{tabular}{|c|c|c|c|c|c|c|}
\hline Variable & Treatment & PRE & Week 8 & POST & Reference Interval & $p$ \\
\hline \multirow[t]{2}{*}{ Systolic BP (mm Hg) } & PLA & $127 \pm 12.0$ & $126.0 \pm 12.2$ & $127.0 \pm 12.2$ & $90-120$ & 0.93 \\
\hline & TRT & $127.6 \pm 12.8$ & $127.7 \pm 8.7$ & $128.0 \pm 10.4$ & & \\
\hline \multirow[t]{2}{*}{ Diastolic BP (mm Hg) } & PLA & $76.1 \pm 9.4$ & $77.0 \pm 8.0$ & $76.4 \pm 9.4$ & $60-80$ & 0.20 \\
\hline & TRT & $76.6 \pm 8.1$ & $74.2 \pm 9.4$ & $78.2 \pm 8.6$ & & \\
\hline \multirow[t]{2}{*}{ Heart Rate (BPM) } & PLA & $68.9 \pm 10.6$ & $70.1 \pm 10.1$ & $70.0 \pm 7.7$ & $<100$ & 0.43 \\
\hline & TRT & $58.9 \pm 12.0$ & $62.1 \pm 9.7$ & $64.9 \pm 9.4$ & & \\
\hline \multirow[t]{2}{*}{ Total Cholesterol (mg/dL) } & PLA & $175.9 \pm 41.9$ & $175.1 \pm 43.2$ & $172.8 \pm 41.0$ & 100-199 & 0.39 \\
\hline & TRT & $161.2 \pm 19.4$ & $173.1 \pm 22.3$ & $164.2 \pm 29.3$ & & \\
\hline \multirow[t]{2}{*}{ Triglycerides (mg/dL) } & PLA & $109.7 \pm 53.0$ & $81.4 \pm 37.9$ & $89.5 \pm 33.3$ & $0-149$ & 0.055 \\
\hline & TRT & $65.9 \pm 22.2$ & $74.5 \pm 33.4$ & $69.3 \pm 36.5$ & & \\
\hline \multirow[t]{2}{*}{ High Density Lipoprotein (mg/dL) } & PLA & $50.1 \pm 13.3$ & $47.6 \pm 12.6$ & $48.5 \pm 11.2$ & $>39$ & 0.38 \\
\hline & TRT & $53.8 \pm 6.4$ & $54.2 \pm 10.2$ & $50.8 \pm 8.2$ & & \\
\hline \multirow[t]{2}{*}{ Very Low Density Lipoprotein (mg/dL) } & PLA & $21.9 \pm 10.5$ & $16.3 \pm 7.6$ & $18.0 \pm 6.8$ & $5-40$ & 0.06 \\
\hline & TRT & $13.3 \pm 4.5$ & $15.0 \pm 6.8$ & $13.8 \pm 7.3$ & & \\
\hline \multirow[t]{2}{*}{ Low Density Lipoprotein (mg/dL) } & PLA & $103.9 \pm 31.7$ & $111.2 \pm 31.2$ & $106.4 \pm 31.0$ & 0-99 & 0.89 \\
\hline & TRT & $94.1 \pm 18.8$ & $103.9 \pm 19.9$ & $99.7 \pm 24.4$ & & \\
\hline
\end{tabular}

Data are presented as mean \pm standard deviation. The $p$-value is derived from an ANOVA and representative of a main effect for group by time

increasing whole-blood ATP levels [31, 32] with a subsequent augmentation of blood flow. ATP and adenosine have been known to induce vasodilation following release from the erythrocytes via production of nitric oxide and prostacyclin $[29,38]$, and it has been recently demonstrated that exogenous ATP supplementation is capable of increasing exercise-induced blood flow [22]. Improved blood flow may increase nutrient delivery. Thus, there is a possibility for a greater effect of circulating amino acids $[9,28]$, glucose [6, 7], and oxygen [10], which may enhance anabolic signaling and/or acute exercise performance, leading to amplified chronic adaptations [10, 35].

The observed changes in FM and \% Fat were likely due to the extended-release caffeine. While an extendedrelease caffeine is yet to be researched, studies have been conducted on caffeine anhydrous and naturallyoccurring caffeine. Caffeine has previously been reported to increase fat oxidation in both lean [25] and overweight individuals [5]. Despite caffeine's effects on metabolism, few studies have investigated the chronic effects of caffeine on body weight and body fat. Sugiura et al. [39] supplemented rats for 4 weeks with caffeine and found caffeine reduced intraperitoneal adipose tissue weights by over $50 \%$ compared to control without a difference in food intake. However, long-term, placebocontrolled human clinical trials do not seem to confirm these results [41] without the addition of tea polyphenols [20], ephedra [41], or a combination of other ingredients [34]. It is also possible that caffeine had an effect on training. Duncan and Oxford have previously reported caffeine increases repetitions performed to failure while reducing perceptions of fatigue [15]. However, in the present study, there were no significant changes in volume performed between groups over time (data not presented). The present study cannot dismiss the potential for caffeine and a blend of ancient peat and apple extracts to have an effect similar to caffeine and tea polyphenols, as phenolic compounds are contained within the ancient peat and apple extracts ingredient blend, though in small amounts $(<1 \%$ efficacious dose $)$ [31], and the combination of caffeine and tea polyphenols have previously been reported to produce reductions in body fat $[19,39]$. Therefore, it may be the combination of caffeine and polyphenols rather than caffeine alone producing this effect, yet it is unlikely at this dose of polyphenols. Finally, the caffeine used in the present study has an extended-release effect due to its binding with pterostilbene, and pterostilbene has been previously reported to reduce BMI in overweight individuals [33].

The primary limitation of this study was the use of several effective ingredients simultaneously. The supplement studied was a multi-ingredient product containing ancient peat, apple extracts, B-vitamins, and an extended-release caffeine containing both caffeine anhydrous and pterostilbene, and while it is possible to speculate on the contributions of each of these ingredients, their individual contributions to the observed effects cannot be definitively interpreted. The present findings are thought to be due, at least in 
Table 4 Hematology Data

\begin{tabular}{|c|c|c|c|c|c|c|}
\hline Variable & Treatment & PRE & Week 8 & POST & Reference Interval & $p$ \\
\hline \multirow[t]{2}{*}{ WBC (x10E3/uL) } & PLA & $5.7 \pm 1.5$ & $5.7 \pm 1.0$ & $5.7 \pm 1.2$ & $3.4-10.8$ & 0.52 \\
\hline & TRT & $5.9 \pm 1.8$ & $5.9 \pm 0.7$ & $6.4 \pm 1.4$ & & \\
\hline \multirow[t]{2}{*}{ RBC (x10E6/uL) } & PLA & $5.3 \pm 0.3$ & $5.4 \pm 0.4$ & $5.4 \pm 0.3$ & $4.14-5.80$ & 0.17 \\
\hline & TRT & $5.3 \pm 0.3$ & $5.4 \pm 0.3$ & $5.3 \pm 0.2$ & & \\
\hline \multirow[t]{2}{*}{ Hemoglobin (g/dL) } & PLA & $16.2 \pm 1.2$ & $16.4 \pm 1.3$ & $16.4 \pm 1.0$ & $12.6-17.7$ & 0.15 \\
\hline & TRT & $16.0 \pm 1.7$ & $16.0 \pm 2.1$ & $15.6 \pm 1.8$ & & \\
\hline \multirow[t]{2}{*}{ Hematocrit (\%) } & PLA & $47.7 \pm 3.0$ & $48.5 \pm 3.3$ & $48.6 \pm 2.7$ & $37.5-51.0$ & 0.31 \\
\hline & TRT & $46.6 \pm 3.6$ & $47.5 \pm 4.9$ & $46.6 \pm 4.3$ & & \\
\hline \multirow[t]{2}{*}{$\mathrm{MCV}(\mathrm{fL})$} & PLA & $89.7 \pm 3.1$ & $89.7 \pm 2.7$ & $89.6 \pm 2.7$ & 79-97 & 0.54 \\
\hline & TRT & $87.5 \pm 7.0$ & $87.9 \pm 7.7$ & $88.3 \pm 8.7$ & & \\
\hline \multirow[t]{2}{*}{$\mathrm{MCH}(\mathrm{pg})$} & PLA & $30.5 \pm 1.1$ & $30.3 \pm 1.0$ & $30.2 \pm 0.9$ & $26.6-33.0$ & 0.77 \\
\hline & TRT & $29.9 \pm 3.2$ & $29.6 \pm 3.5$ & $29.6 \pm 3.5$ & & \\
\hline \multirow[t]{2}{*}{$\mathrm{MCHC}(\mathrm{g} / \mathrm{dL})$} & PLA & $34.0 \pm 1.0$ & $33.8 \pm 0.6$ & $33.7 \pm 0.7$ & $31.5-35.7$ & 0.63 \\
\hline & TRT & $34.2 \pm 1.6$ & $33.6 \pm 1.6$ & $33.5 \pm 1.1$ & & \\
\hline \multirow[t]{2}{*}{ RDW (\%) } & PLA & $13.6 \pm 0.5$ & $13.4 \pm 0.4$ & $13.4 \pm 0.4$ & $12.3-15.4$ & 0.06 \\
\hline & TRT & $13.6 \pm 1.2$ & $13.9 \pm 1.8$ & $13.7 \pm 1.7$ & & \\
\hline \multirow[t]{2}{*}{ Platelets (x10E3/uL) } & PLA & $236.7 \pm 29.1$ & $239.4 \pm 45.4$ & $241.6 \pm 31.6$ & $155-379$ & 0.61 \\
\hline & TRT & $274.5 \pm 61.0$ & $286.4 \pm 77.6$ & $273.7 \pm 79.5$ & & \\
\hline \multirow[t]{2}{*}{ Neutrophils (\%) } & PLA & $53.5 \pm 8.3$ & $49.0 \pm 6.7$ & $49.5 \pm 9.1$ & $40-74$ & 0.23 \\
\hline & TRT & $52.6 \pm 12.2$ & $50.4 \pm 10.7$ & $55.5 \pm 10.9$ & & \\
\hline \multirow[t]{2}{*}{ Lymphs (\%) } & PLA & $35.1 \pm 6.9$ & $38.9 \pm 6.5$ & $38.2 \pm 8.3$ & $14-46$ & 0.15 \\
\hline & TRT & $34.3 \pm 10.6$ & $35.9 \pm 9.0$ & $30.8 \pm 8.9$ & & \\
\hline \multirow[t]{2}{*}{ Monocytes (\%) } & PLA & $8.7 \pm 1.8$ & $9.4 \pm 2.1$ & $9.4 \pm 2.4$ & $4-12$ & 0.77 \\
\hline & TRT & $9.7 \pm 2.0$ & $10.0 \pm 1.9$ & $10.8 \pm 3.6$ & & \\
\hline \multirow[t]{2}{*}{ Eos (\%) } & PLA & $2.1 \pm 1.2$ & $2.4 \pm 2.4$ & $2.5 \pm 2.3$ & $0-5$ & 0.27 \\
\hline & TRT & $2.8 \pm 2.4$ & $3.0 \pm 2.4$ & $2.3 \pm 1.6$ & & \\
\hline \multirow[t]{2}{*}{ Basos (\%) } & PLA & $0.6 \pm 0.7$ & $0.4 \pm 0.7$ & $0.4 \pm 0.7$ & $0-3$ & 0.43 \\
\hline & TRT & $0.6 \pm 0.7$ & $0.7 \pm 0.7$ & $0.5 \pm 0.7$ & & \\
\hline \multirow[t]{2}{*}{ Neutrophils (Absolute) (x10E3/uL) } & PLA & $3.1 \pm 1.4$ & $2.8 \pm 0.8$ & $2.9 \pm 1.0$ & $1.4-7.0$ & 0.41 \\
\hline & TRT & $3.2 \pm 1.6$ & $3.0 \pm 0.7$ & $3.6 \pm 1.4$ & & \\
\hline \multirow[t]{2}{*}{ Lymphs (Absolute) (x10E3/uL) } & PLA & $1.9 \pm 0.1$ & $2.2 \pm 0.3$ & $2.1 \pm 0.4$ & $0.7-3.1$ & 0.33 \\
\hline & TRT & $1.9 \pm 0.3$ & $2.1 \pm 0.5$ & $1.9 \pm 0.5$ & & \\
\hline \multirow[t]{2}{*}{ Monocytes (Absolute) (x10E3/uL) } & PLA & $0.5 \pm 0.2$ & $0.5 \pm 0.1$ & $0.5 \pm 0.2$ & $0.1-0.9$ & 0.52 \\
\hline & TRT & $0.6 \pm 0.2$ & $0.6 \pm 0.1$ & $0.7 \pm 0.2$ & & \\
\hline \multirow[t]{2}{*}{ Eos (Absolute) (x10E3/uL) } & PLA & $0.1 \pm 0.1$ & $0.1 \pm 0.1$ & $0.1 \pm 0.1$ & $0.0-0.4$ & 0.65 \\
\hline & TRT & $0.2 \pm 0.1$ & $0.2 \pm 0.1$ & $0.2 \pm 0.1$ & & \\
\hline \multirow[t]{2}{*}{ Baso (Absolute) (x10E3/uL) } & PLA & $0.0 \pm 0.0$ & $0.0 \pm 0.0$ & $0.0 \pm 0.0$ & $0.0-0.2$ & 0.10 \\
\hline & TRT & $0.0 \pm 0.1$ & $0.0 \pm 0.0$ & $0.0 \pm 0.0$ & & \\
\hline
\end{tabular}

Data are presented as mean \pm standard deviation. The $p$-value is derived from an ANOVA and representative of a main effect for group by time

part, to the elevation of whole-blood or intramuscular ATP levels based on the results reported by ReyesIzquierdo et al. on acute administration of the proprietary blend of ancient peat and apple extracts [31, 32]. However, this effect may not persist in a chronic setting, and the present study did not measure wholeblood or intramuscular ATP levels at any time point. The current study also did not feature a nonexercising control group, so the effects of the training program are unable to be determined. 
Table 5 Blood Chemistry Data. Data are presented as mean \pm standard deviation

\begin{tabular}{|c|c|c|c|c|c|c|}
\hline Variable & Treatment & PRE & Week 8 & POST & Reference Interval & $p$ \\
\hline \multirow[t]{2}{*}{ Serum Glucose (mg/dL) } & PLA & $90.5 \pm 11.0$ & $89.3 \pm 3.6$ & $90.2 \pm 5.4$ & $65-99$ & 0.84 \\
\hline & TRT & $88.8 \pm 6.1$ & $89.6 \pm 7.0$ & $90.0 \pm 6.8$ & & \\
\hline \multirow[t]{2}{*}{$\mathrm{BUN}(\mathrm{mg} / \mathrm{dL})$} & PLA & $17.3 \pm 4.9$ & $17.8 \pm 3.9$ & $17.5 \pm 4.2$ & $6-20$ & 0.98 \\
\hline & TRT & $15.1 \pm 3.2$ & $15.7 \pm 3.1$ & $15.7 \pm 3.5$ & & \\
\hline \multirow[t]{2}{*}{ Serum Creatinine (mg/dL) } & PLA & $0.97 \pm 0.12$ & $1.04 \pm 0.12^{\mathrm{a}}$ & $1.13 \pm 0.17^{* a b}$ & $0.76-1.27$ & 0.001 \\
\hline & TRT & $1.05 \pm 0.15$ & $1.10 \pm 0.11$ & $1.05 \pm 0.12^{* \neq}$ & & \\
\hline \multirow[t]{2}{*}{ eGFR (mL/min/1.73) } & PLA & $105.6 \pm 12.9$ & $98.7 \pm 12.5^{a}$ & $91.0 \pm 14.9^{a}$ & $>59$ & 0.01 \\
\hline & TRT & $101.0 \pm 15.8$ & $95.2 \pm 11.1$ & $100.3 \pm 12.9^{* \neq}$ & & \\
\hline \multirow[t]{2}{*}{ BUN/Creatinine Ratio } & PLA & $18.0 \pm 5.9$ & $17.3 \pm 4.0$ & $15.8 \pm 4.4$ & $8-19$ & 0.33 \\
\hline & TRT & $14.4 \pm 3.1$ & $14.3 \pm 1.8$ & $15.0 \pm 3.7$ & & \\
\hline \multirow[t]{2}{*}{ Serum Sodium (mmol/L) } & PLA & $138.8 \pm 1.8$ & $139.6 \pm 1.3$ & $140.4 \pm 2.1$ & $134-144$ & 0.43 \\
\hline & TRT & $138.9 \pm 1.7$ & $138.7 \pm 1.3$ & $140.4 \pm 1.3$ & & \\
\hline \multirow[t]{2}{*}{ Serum Potassium (mmol/L) } & PLA & $4.4 \pm 0.4$ & $4.5 \pm 0.5$ & $4.3 \pm 0.3$ & $3.5-5.2$ & 0.06 \\
\hline & TRT & $4.4 \pm 0.3$ & $4.4 \pm 0.3$ & $4.6 \pm 0.3$ & & \\
\hline \multirow[t]{2}{*}{ Serum Chloride (mmol/L) } & PLA & $102.1 \pm 1.6$ & $101.0 \pm 1.4$ & $102.4 \pm 2.2$ & $97-108$ & 0.92 \\
\hline & TRT & $101.7 \pm 1.8$ & $100.7 \pm 2.1$ & $102.3 \pm 1.6$ & & \\
\hline \multirow[t]{2}{*}{ Carbon Dioxide (mmol/L) } & PLA & $23.6 \pm 2.4$ & $22.1 \pm 1.5$ & $22.5 \pm 1.5$ & $19-28$ & 0.24 \\
\hline & TRT & $23.1 \pm 2.4$ & $21.0 \pm 1.2$ & $22.8 \pm 1.4$ & & \\
\hline \multirow[t]{2}{*}{ Serum Calcium (mg/dL) } & PLA & $9.4 \pm 0.4$ & $9.4 \pm 0.3$ & $9.3 \pm 0.3$ & $8.7-10.2$ & 0.45 \\
\hline & TRT & $9.6 \pm 0.4$ & $9.5 \pm 0.3$ & $9.4 \pm 0.4$ & & \\
\hline \multirow[t]{2}{*}{ Serum Protein (g/dL) } & PLA & $7.1 \pm 0.3$ & $6.9 \pm 0.3$ & $6.9 \pm 0.3$ & $6.0-8.5$ & 0.58 \\
\hline & TRT & $7.2 \pm 0.3$ & $6.9 \pm 0.2$ & $6.9 \pm 0.3$ & & \\
\hline \multirow[t]{2}{*}{ Serum Albumin (g/dL) } & PLA & $4.4 \pm 0.3$ & $4.6 \pm 0.2$ & $4.6 \pm 0.2$ & $3.5-5.5$ & 0.65 \\
\hline & TRT & $4.5 \pm 0.2$ & $4.6 \pm 0.2$ & $4.6 \pm 0.2$ & & \\
\hline \multirow[t]{2}{*}{ Globulin (g/dL) } & PLA & $2.6 \pm 0.2$ & $2.3 \pm 0.2$ & $2.3 \pm 0.2$ & $1.5-4.5$ & 0.83 \\
\hline & TRT & $2.7 \pm 0.2$ & $2.3 \pm 0.3$ & $2.3 \pm 0.3$ & & \\
\hline \multirow[t]{2}{*}{ Albumin:Globulin Ratio } & PLA & $1.7 \pm 0.2$ & $2.0 \pm 0.2$ & $2.0 \pm 0.3$ & $1.1-2.5$ & 0.76 \\
\hline & TRT & $1.7 \pm 0.2$ & $2.0 \pm 0.3$ & $2.1 \pm 0.3$ & & \\
\hline \multirow[t]{2}{*}{ Bilirubin (mg/dl) } & PLA & $0.6 \pm 0.2$ & $0.6 \pm 0.2$ & $0.6 \pm 0.2$ & $0.0-1.2$ & 0.06 \\
\hline & TRT & $0.6 \pm 0.3$ & $0.7 \pm 0.4$ & $0.5 \pm 0.2$ & & \\
\hline \multirow[t]{2}{*}{ Alkaline Phosphatase (IU/L) } & PLA & $76.4 \pm 13.8$ & $81.3 \pm 14.6$ & $79.9 \pm 16.1$ & $39-117$ & 0.53 \\
\hline & TRT & $70.5 \pm 20.8$ & $79.5 \pm 27.2$ & $77.8 \pm 23.4$ & & \\
\hline \multirow[t]{2}{*}{ AST (IU/L) } & PLA & $25.5 \pm 7.8$ & $26.9 \pm 7.4$ & $25.8 \pm 8.9$ & $0-40$ & 0.81 \\
\hline & TRT & $25.1 \pm 7.5$ & $28.9 \pm 11.6$ & $27.2 \pm 12.7$ & & \\
\hline \multirow[t]{2}{*}{$\operatorname{ALT}(I U / L)$} & PLA & $23.7 \pm 4.9$ & $24.3 \pm 7.0$ & $22.5 \pm 6.3$ & $0-44$ & 0.48 \\
\hline & TRT & $23.5 \pm 11.1$ & $23.3 \pm 8.6$ & $26.5 \pm 12.2$ & & \\
\hline
\end{tabular}

*indicates significantly different from PLA at the corresponding time point. $\neq$ indicates significantly different from PLA at week 8 . The $\mathrm{p}$-value is derived from an ANOVA and representative of a main effect for group by time. Significant within-group time differences are indicated by ${ }^{a}$ (different from pre) and ${ }^{b}$ (different from week 8 ) for variables with a significant group $x$ time interaction

\section{Conclusions}

This is the first study to examine the effects of an ATPenhancing supplement combined with an extendedrelease caffeine on body composition. There have been no previously published investigations regarding an extended-release caffeine, and there is a paucity of studies conducted on the long-term effects of caffeine on body composition without additional tea polyphenols. The combination of these ingredients appear to beneficially augment body composition when consumed 


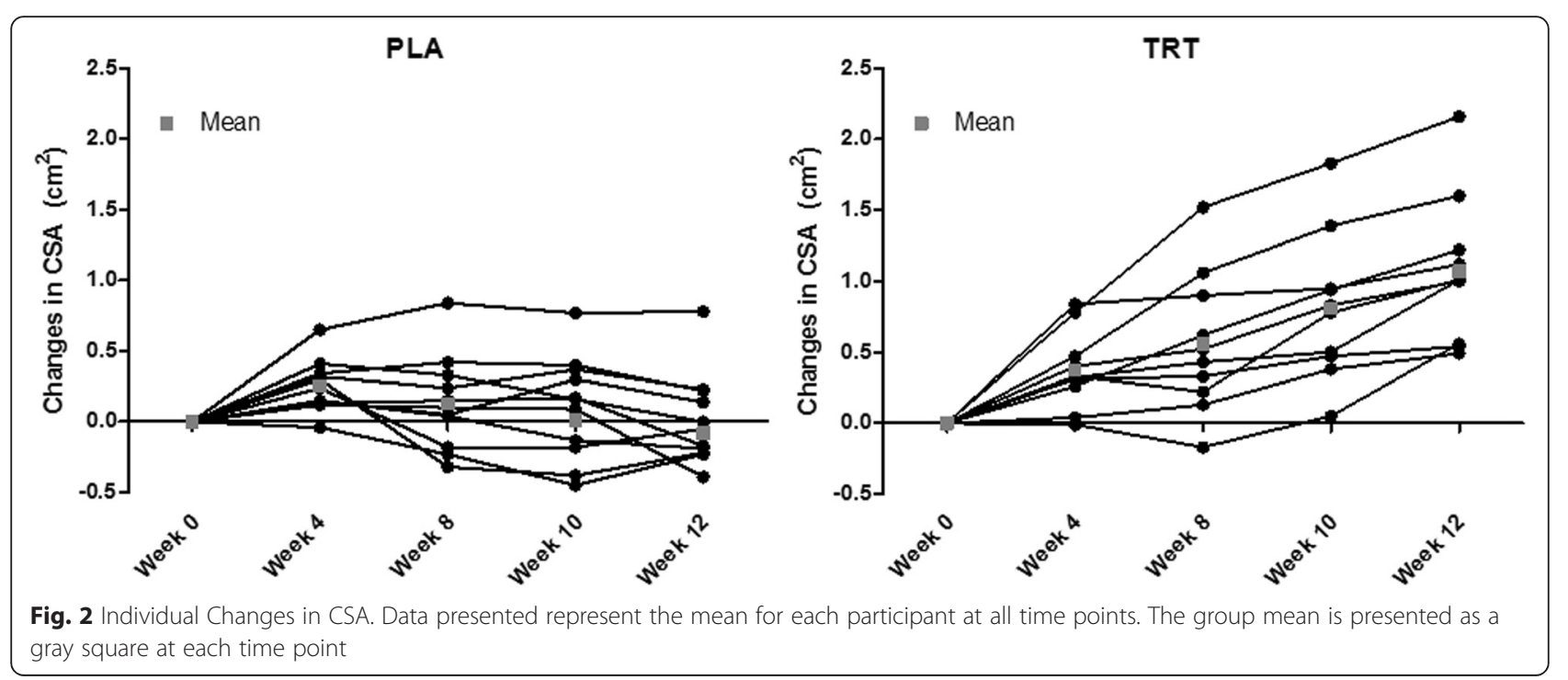

during a periodized resistance training protocol. Increases in measures of muscle mass were likely produced by the blend of ancient peat and apple extracts, and the small reduction of body fat observed in the TRT group, versus an increase in the PLA group, is presumably due to the caffeine, although, a synergistic effect of these ingredients cannot be entirely dismissed from the present investigation. Athletes who benefit from increased muscle mass but not fat mass, such as bodybuilders and skill-position football players, as well as recreational athletes seeking improved body composition may benefit from the use of the present supplement combination. Future research should examine the extended-release caffeine used in the present study as a standalone supplement in conjunction with an exercise protocol designed for body fat reduction to determine its potential for reducing or maintaining body fat. Moreover, future research may be interested in exploring the effects of ancient peat and apple extracts in an endurance setting.

\section{Abbreviations}

$\%$ Fat, percent fat; A/G, albumin to globulin ratio; ALT, alanine aminotransferase ; ANOVA, analysis of variance; AST, aspartate aminotransferase; ATP, adenosine triphosphate; BUN, blood urea nitrogen; CSA, cross-sectional area; DEXA, dual emissions x-ray absorptiometry; eGFR, estimated glomerular filtration rate; FM, fat mass; $F$, fat thickness; HDL, high-density lipoprotein; LDL, low density lipoprotein; LST, lean soft tissue; MCH, mean corpuscular hemoglobin; MCHC, mean corpuscular hemoglobin concentration; MCV, mean corpuscular volume; MT, muscle thickness; PLA, placebo; RBC, red blood cell count; RDW, red blood cell distribution width; TRT, treatment; VLDL, very low density lipoprotein; WBC, white blood cell count

\section{Acknowledgements}

The authors thank Aaron Tribby, Schyler Tabor, Dylan Lefever, Chadwick Hughes, and J. Daniel Griffin for their assistance in monitoring study participants' compliance.

\section{Funding}

The authors would like to thank VDF FutureCeuticals Inc. for funding this investigation.

\section{Availability of data and materials}

Raw data is presently unavailable for sharing due to the agreement between the researching parties and funding organizations.

\section{Authors' contributions}

All authors contributed to the conception of the experimental design and drafting of the manuscript. JJ, RV, PF, and MM participated in data collection. Data were analyzed by JJ and JM. All authors have read and approved the final version of the manuscript.

\section{Competing interests}

This research took place at the MusclePharm Sports Science Institute. JJ, RV, JM, PF, MM, and MK were employees of MusclePharm Corporation at the time of the investigation, which has since included at least one of these ingredients in a product. JJ, RV, JM, and MM were not affiliated with MusclePharm Corporation during the drafting of the manuscript. Additionally, this manuscript should not be viewed as endorsement by the investigators, Texas Woman's University, Maximum Mobile Fitness, the American Public University, or MusclePharm Corporation.

\section{Author details}

'Department of Nutrition and Food Sciences, Texas Woman's University, 401 AME Drive \#7101, Denton, TX 76207, USA. ${ }^{2}$ American Public University System, School of Health Sciences, Charles Town, WV, USA. ${ }^{3}$ MusclePharm Sports Science Institute, MusclePharm Corp., Denver, CO, USA. ${ }^{4}$ Maximum Mobile Fitness, Spearfish, SD, USA.

Received: 22 March 2016 Accepted: 1 June 2016

Published online: 10 June 2016

\section{References}

1. Acheson KJ, Zahorska-Markiewicz B, Pittet P, Anantharaman K, Jequier E. Caffeine and coffee: their influence on metabolic rate and substrate utilization in normal weight and obese individuals. Am J Clin Nutr. 1980;33:989-97.

2. Agteresch HJ, Dagnelie PC, van den Berg JW, Wilson JH. Adenosine triphosphate: established and potential clinical applications. Drugs. 1999;58:211-32.

3. Alessio HM, Hagerman AE, Fulkerson BK, Ambrose J, Rice RE, Wiley RL. Generation of reactive oxygen species after exhaustive aerobic and isometric exercise. Med Sci Sports Exerc. 2000;32:1576-81.

4. Arts IC, Coolen EJ, Bours MJ, Huyghebaert N, Stuart MA, Bast A, Dagnelie PC Adenosine 5'-triphosphate (ATP) supplements are not orally bioavailable: a 
randomized, placebo-controlled cross-over trial in healthy humans. J Int Soc Sports Nutr. 2012;9:16.

5. Astrup A, Toubro S, Cannon S, Hein P, Breum L, Madsen J. Caffeine: a double-blind, placebo-controlled study of its thermogenic, metabolic, and cardiovascular effects in healthy volunteers. Am J Clin Nutr. 1990;51:759-67.

6. Baron $A D$, Clark MG. Role of blood flow in the regulation of muscle glucose uptake. Annu Rev Nutr. 1997;17:487-99.

7. Baron AD, Steinberg H, Brechtel G, Johnson A. Skeletal muscle blood flow independently modulates insulin-mediated glucose uptake. Am J Physiol. 1994:266:E248-53.

8. Beck TW, Housh TJ, Schmidt RJ, Johnson GO, Housh DJ, Coburn JW, Malek MH. The acute effects of a caffeine-containing supplement on strength, muscular endurance, and anaerobic capabilities. J Strength Cond Res. 2006;20:506-10.

9. Bohe J, Low A, Wolfe RR, Rennie MJ. Human muscle protein synthesis is modulated by extracellular, not intramuscular amino acid availability: a dose-response study. J Physiol. 2003:552:315-24.

10. Buchheit M, Cormie P, Abbiss CR, Ahmaidi S, Nosaka KK, Laursen PB. Muscle deoxygenation during repeated sprint running: Effect of active vs. passive recovery. Int J Sports Med. 2009;30:418-25.

11. Burnstock $G$, Knight $G E$, Greig AV. Purinergic signaling in healthy and diseased skin. J Invest Dermatol. 2012;132:526-46.

12. Chang JC, Kou SJ, Lin WT, Liu CS. Regulatory role of mitochondria in oxidative stress and atherosclerosis. World J Cardiol. 2010;2:150-9.

13. Coolen EJ, Arts IC, Bekers O, Vervaet C, Bast A, Dagnelie PC. Oral bioavailability of ATP after prolonged administration. Br J Nutr. 2011;105:357-66.

14. Desbrow B, Biddulph C, Devlin B, Grant GD, Anoopkumar-Dukie S, Leveritt MD. The effects of different doses of caffeine on endurance cycling time trial performance. J Sports Sci. 2012;30:115-20.

15. Duncan MJ, Oxford SW. The effect of caffeine ingestion on mood state and bench press performance to failure. J Strength Cond Res. 2011;25:178-85.

16. Farney TM, McCarthy CG, Canale RE, Allman Jr RJ, Bloomer RJ. Hemodynamic and hematologic profile of healthy adults ingesting dietary supplements containing 1,3-dimethylamylamine and caffeine. Nutr Metab Insights. 2012;5:1-12

17. Goldstein ER, Ziegenfuss T, Kalman D, Kreider R, Campbell B, Wilborn C, Taylor L, Willoughby D, Stout J, Graves BS, Wildman R, Ivy JL, Spano M, Smith AE, Antonio J. International society of sports nutrition position stand: caffeine and performance. J Int Soc Sports Nutr. 2010;7:5.

18. Herda TJ, Ryan ED, Stout JR, Cramer JT. Effects of a supplement designed to increase ATP levels on muscle strength, power output, and endurance. J Int Soc Sports Nutr. 2008;5:3.

19. Hursel R, Viechtbauer W, Westerterp-Plantenga MS. The effects of green tea on weight loss and weight maintenance: a meta-analysis. Int J Obes (Lond). 2009;33:956-61.

20. Hursel R, Westerterp-Plantenga MS. Thermogenic ingredients and body weight regulation. Int J Obes (Lond). 2010;34:659-69.

21. Jackman M, Wendling P, Friars D, Graham TE. Metabolic catecholamine, and endurance responses to caffeine during intense exercise. J Appl Physiol (1985). 1996;81:1658-63.

22. Jager R, Roberts MD, Lowery RP, Joy JM, Cruthirds CL, Lockwood CM, Rathmacher JA, Purpura M, Wilson JM. Oral adenosine-5'-triphosphate (ATP) administration increases blood flow following exercise in animals and humans. J Int Soc Sports Nutr. 2014;11:28.

23. Jordan AN, Jurca R, Abraham EH, Salikhova A, Mann JK, Morss GM, Church TS, Lucia A, Earnest CP. Effects of oral ATP supplementation on anaerobic power and muscular strength. Med Sci Sports Exerc. 2004;36:983-90.

24. Joy JM, Falcone PH, Vogel RM, Mosman MM, Kim MP, Moon JR. Supplementation with a proprietary blend of ancient peat and apple extract may improve body composition without affecting hematology in resistance-trained men. Appl Physiol Nutr Metab. 2015;40(11):1171-7.

25. Keijzers GB, De Galan BE, Tack CJ, Smits P. Caffeine can decrease insulin sensitivity in humans. Diabetes Care. 2002;25:364-9.

26. Kushmerick MJ, Conley KE. Energetics of muscle contraction: the whole is less than the sum of its parts. Biochem Soc Trans. 2002;30:227-31.

27. Lieberman HR, Tharion WJ, Shukitt-Hale B, Speckman KL, Tulley R. Effects of caffeine, sleep loss, and stress on cognitive performance and mood during U.S. Navy SEAL training. Sea-Air-Land. Psychopharmacology (Berl). 2002; 164:250-61

28. Morgan HE, Earl DC, Broadus A, Wolpert EB, Giger KE, Jefferson LS. Regulation of protein synthesis in heart muscle. I. Effect of amino acid levels on protein synthesis. J Biol Chem. 1971;246:2152-62.
29. Nyberg M, Mortensen SP, Thaning P, Saltin B, Hellsten Y. Interstitial and plasma adenosine stimulate nitric oxide and prostacyclin formation in human skeletal muscle. Hypertension. 2010;56:1102-8.

30. Rathmacher JA, Fuller Jr JC, Baier SM, Abumrad NN, Angus HF, Sharp RL. Adenosine-5'-triphosphate (ATP) supplementation improves low peak muscle torque and torque fatigue during repeated high intensity exercise sets. J Int Soc Sports Nutr. 2012;9:48.

31. Reyes-Izquierdo T, Nemzer B, Argumedo R, Shu C, Huynh L, Pietrzkowski Z. Effect of the dietary supplement ElevATP on blood ATP level: An acute pilot clinical study. J Aging Res Clin Practice. 2013;2:178-84.

32. Reyes-Izquierdo T, Shu C, Argumedo R, Nemzer B, Pietrzkowski Z. The effect of elevATP'm on whole blood ATP levels: a single dose, crossover clinical study. J Aging Res Clin Practice. 2014;3:56-60.

33. Riche DM, Riche KD, Blackshear CT, McEwen CL, Sherman JJ, Wofford MR, Griswold ME. Pterostilbene on metabolic parameters: a randomized, doubleblind, and placebo-controlled trial. Evid Based Complement Alternat Med. 2014;2014:459165.

34. Roberts MD, Dalbo VJ, Hassell SE, Stout JR, Kerksick CM. Efficacy and safety of a popular thermogenic drink after 28 days of ingestion. J Int Soc Sports Nutr. 2008:5:19.

35. Rodriguez NR, DiMarco NM, Langley S. Nutrition and athletic performance. Med Sci Sports Exerc. 2009:41:709-31.

36. Schneiker KT, Bishop D, Dawson B, Hackett LP. Effects of caffeine on prolonged intermittent-sprint ability in team-sport athletes. Med Sci Sports Exerc. 2006;38:578-85.

37. Schubert MM, Hall S, Leveritt M, Grant G, Sabapathy S, Desbrow B. Caffeine consumption around an exercise bout: effects on energy expenditure, energy intake, and exercise enjoymen. J Appl Physiol (1985). 2014;117:745-54.

38. Sprague RS, Bowles EA, Achilleus D, Ellsworth ML. Erythrocytes as controllers of perfusion distribution in the microvasculature of skeletal muscle. Acta Physiol (Oxf). 2011;202:285-92.

39. Sugiura C, Nishimatsu S, Moriyama T, Ozasa S, Kawada T, Sayama K. Catechins and Caffeine Inhibit Fat Accumulation in Mice through the Improvement of Hepatic Lipid Metabolism. J Obes. 2012;2012:520510.

40. Swamy MS, Sivanna N, Tamatam A, Khanum F. Effect of poly phenols in enhancing the swimming capacity of rats. Funct Foods Health Dis. 2011;1:482-91.

41. Toubro S, Astrup AV, Breum L, Quaade F. Safety and efficacy of long-term treatment with ephedrine, caffeine and an ephedrine/caffeine mixture. Int J Obes Relat Metab Disord. 1993;17 Suppl 1:S69-72.

42. Vogel RM, Joy JM, Falcone PH, Mosman MM, Kim MP, Moon JR. Safety of a dose-escalated pre-workout supplement in recreationally active females. J Int Soc Sports Nutr. 2015;12:12.

43. Weir JP. Quantifying test-retest reliability using the intraclass correlation coefficient and the SEM. J Strength Cond Res. 2005;19:231-40.

44. Wilson JM, Joy JM, Lowery RP, Roberts MD, Lockwood CM, Manninen AH, Fuller JC, De Souza EO, Baier SM, Wilson SM, Rathmacher JA. Effects of oral adenosine-5'-triphosphate supplementation on athletic performance, skeletal muscle hypertrophy and recovery in resistance-trained men. Nutr Metab (Lond). 2013;10:57.

\section{Submit your next manuscript to BioMed Central and we will help you at every step:}

- We accept pre-submission inquiries

- Our selector tool helps you to find the most relevant journal

- We provide round the clock customer support

- Convenient online submission

- Thorough peer review

- Inclusion in PubMed and all major indexing services

- Maximum visibility for your research

Submit your manuscript at www.biomedcentral.com/submit
) Biomed Central 